\title{
Estradiol, SHBG and leptin interplay with food craving and intake across the menstrual cycle
}

\author{
Sridevi Krishnan ${ }^{1}$, Rebecca R Tryon ${ }^{1}$, William F Horn ${ }^{2}$, Lucas Welch ${ }^{1}$, Nancy L Keim ${ }^{1,2}$
}

1 - Department of Nutrition, University of California Davis CA

2 - USDA, ARS, Western Human Nutrition Research Center, Obesity and Metabolism Research Unit, 430 West

Health Sciences Dr., Davis, CA 95616

Running head: Food craving behaviors in the menstrual cycle

Corresponding author: Nancy L. Keim, USDA, ARS, Western Human Nutrition Research Center, 430 West

Health Sciences Drive, Davis, CA 95616. 530-752-4163 (Phone), 530-752-5695 (FAX), email:

nancy.keim@ars.usda.gov

Funding: This study was funded by USDA Project 5306-51530-019-00D, 2032-51530-022-00D, and Jastro

Shields award from UC Davis. The U.S. Department of Agriculture is an equal opportunity provider and employer.

Acknowledgements: The authors would like to thank Emma While, Evelyn Holguin, Jerome Crawford, Leslie Woodhouse, Joseph Domek, Adrianne Widaman, Ellen Bonnel, Dustin Burnett, Sara Stoffel, Julie Edwards for assistance with this project.

\section{Clinical Trial Registration Number: NCT01407692}




\section{ABSTRACT}

Objective: To understand the association between ovarian hormones, non-acute satiety hormones and craving calorie dense foods in the luteal phase. Methods: 17 premenopausal women, mean age 23.2 y, mean BMI 22.4 $\mathrm{kg} / \mathrm{m}^{2}$ with regular menstrual cycles were studied during late follicular (FP) and luteal phases (LP). Estradiol, progesterone, DHEAS, SHBG, insulin and leptin, were measured in fasting samples. The validated Food Craving Inventory was used to record the types of foods volunteers habitually ate - rich in fat, carbohydrate or sweet taste, as well as craved during the LP of their menstrual cycle. Results: Estradiol was inversely associated with leptin in FP $(r=-0.62, p=0.01)$. Leptin was inversely associated with habitual intake of sweet foods, in both phases (FP: $r$ $=-0.64, p=0.01 ; \mathrm{LP}: \mathrm{r}=-0.63, \mathrm{p}=0.01)$. SHBG in LP was positively associated with craving sweet and carbohydrate rich foods. Hierarchical cluster analysis revealed two groups of women, one with high estradiol, high estradiol/leptin ratio, high sweet and carbohydrate cravings $(\mathrm{p}<0.05)$; the other group had lower estradiol, lower estradiol/leptin ratio, and reported less craving. Conclusions: The estradiol-leptin axis may be a determinant of luteal phase craving and habitual food intake in menstruating women.

Key Words: Leptin, estradiol, food intake, sex hormones, craving. 
Appetite regulation and energy intake fluctuate with phases of the menstrual cycle [1], and it is

3 recommended that the cycle phases be considered for dietary intervention studies [2]. Positive energy balance in

4 the luteal phase, compared to a more negative or energy balance during the follicular phase has been reported in

5 lean women [3, 4]. In addition, food cravings for sweet tasting, carbohydrate- and fat-rich foods have been

6 linked to the luteal phase of the menstrual cycle $[5,6]$. These food cravings may be associated with increased

7 energy intake [6]. Since food cravings may be crucial to managing obesity and its treatment [7], it is highly

8 relevant to understand if food cravings are associated with menstrual cycle hormones [8].

Estradiol and progesterone influence food intake behaviors in animals [9]. Estradiol reduces food intake in rats $[10,11]$ via activation of estrogen receptor $\alpha$ [12]. The effect of progesterone has been unclear; some

11 evidence suggests it enhances [9], while other evidence suggests it antagonizes the anorexigenic effect of estradiol [13]. Estradiol has also shown to increase sucrose taste threshold in rats [14]. The satiety hormone

13 leptin has been shown to reduce sweet taste sensitivity in rats, thereby affecting palatability, and subsequent 14 behavioral responses [15]. Limited human studies yield conflicting results; leptin appears to be positively 15 associated with stress-induced sweet-craving in one study [16], while in another study higher leptin is 16 associated with preference for lower sugar intake in obese women [17]. Interestingly, leptin has been shown to 17 increase at peak follicular and luteal phase, alongside estradiol and progesterone, in young women (18 - 30y 18 age) [18]. Researchers speculate that estradiol likely increases leptin secretion in humans [19], and animal 19 studies suggest a similar role [20]. While estradiol is necessary for leptin action in the hypothalamus [21], the 20 anorexigenic effect of estradiol is independent of estradiol's modulation of leptin [22]. A study looking at food 21 intake, body weight and preference for sweet tasting food across the menstrual cycle noted that there was 22 increase in both food intake and body weight in the luteal phase, compared to the follicular phase, but that the 23 sweet preference was significantly lower in the luteal than the follicular phase [23]. The luteal phase increase in 24 body weight could be due to increased blood volume, but the reduced preference for sweet seems contrary to 
the reported increase in food intake. However, this study did not measure leptin concentrations, nor did it report other factors that can influence satiety measures, such as insulin.

Based on animal literature, estradiol reduces food intake, and it is unclear whether progesterone enhances or antagonizes this. While estradiol and leptin have a positive association with each other, and can be viewed as anorexigenic hormones, their impact on eating and craving behaviors in humans is unclear. It is possible to speculate that estradiol in the unopposed state (follicular phase) reduces food intake, but when opposed by progesterone (luteal phase), estradiol has diminished impact. However, this needs to be tested and established.

Given that high sweet/fat/carbohydrate craving and food intake appear to increase during the luteal phase of the menstrual cycle, the consideration that both estradiol and progesterone may be anorexigenic is probably unlikely. While several studies have looked at craving and binge eating behaviors in women with eating disorders or pre-menstrual dysphoric disorder (PMDD), the association between ovarian hormones and craving in healthy women is not well established. Further, as pointed out by Davidsen et al no menstrual cycle study has examined food intake and cravings to associate these behaviors with estradiol and leptin [24]. The current study addressed the following questions: Does circulating leptin concentration alter craving of sweettasting and carbohydrate-rich foods in the luteal phase? Are cravings associated with circulating estradiol and progesterone? The follicular phase of the study is associated with low estradiol and progesterone concentrations (i.e. conducive to metabolic testing), while the luteal phase is marked by higher circulating ovarian steroid hormones [25]. Since the physiological and behavioral impacts of the phases are a function of their circulating ovarian hormones, we decided to choose the time to test women based on their peak concentrations, hence, we chose to study estradiol at its peak (i.e. late follicular phase, close to ovulation), and estradiol and progesterone at their peak in the luteal phase (mid-late luteal phase). We hypothesized that leptin would be associated with reduced cravings as well as reduced intake of sweet-tasting and carbohydrate rich foods. Moreover, we also hypothesized that we could determine whether women are "high cravers" or "low cravers" based on their ovarian and leptin hormone concentrations. 
53 METHODS

54 Subject screening: Women with regular menstrual cycles between the ages of 18-30y, with BMI between 18-

$5525 \mathrm{~kg} / \mathrm{m}^{2}$ were recruited and screened for eligibility. A modified menstrual cycle history questionnaire (SWAN 56 study-form A and menstrual calendar) [26] was administered using Survey Monkey® (SurveyMonkey.com,

57 LLC). Women, who reported regular menstrual cycles (26-38 days), did not use hormonal contraceptives, had 58 no significant weight change $( \pm 5 \%)$ in the past 6 months, and were nulliparous were enrolled. A history of 59 hypertension, cardiovascular disease, prediabetes, type 2 diabetes, or dyslipidemia were exclusionary criteria for 60 this study. At screening, which happened at random times irrespective of their menstrual cycle, a fasting blood 61 draw (8h overnight fast) was used to evaluate fasting blood glucose and lipid concentrations, body weight was 62 measured in light clothing using a Tanita BWB 627A Class III electronic scale, and height was measured using 63 a wall-mounted, Ayrton Stadiometer (Model S100, Ayrton Corp., Prior Lake, MN). This study was approved by 64 the Institutional Review Board of the University of California Davis, and all participants reviewed and signed 65 the study consent form. At screening, participants completed a 34-item food inventory that consisted of the 66 same foods from the Food Craving Inventory (FCI - see Food Craving Inventory section below for more 67 details) [27] to capture habitual intake of sweet, high fat, carbohydrate, fast food and sweet beverages. They 68 were asked to reflect back on their habitual intake of these foods between $2 \mathrm{pm}$ and bedtime in the three months 69 preceding this visit, and respond based on their recollection. In addition, participants also answered the three70 factor eating questionnaire (TFEQ) [28]. The TFEQ consists of 51 questions, 21 of which address cognitive 71 restraint (scored $0-21), 16$ address disinhibition (scored $0-16)$, and 14 (scored $0-14$ ) susceptibility to 72 hunger. For all categories, the scores were summed, and a higher score denoted higher levels of restrained 73 eating, disinhibition and susceptibility to hunger.

Tracking the menstrual cycle: Enrolled participants were given a menstrual cycle calendar, and a set of five 76 urinary LH test kits (One Step Strip Style LH urine ovulation test, Access Medical Products LLC, GA), with 
training on how to use these appropriately. They were instructed to report the first day of their bleeding phase, at which point, they were instructed to begin their menstrual cycle calendar to track their cycle. Dates of the previous 3 menstrual cycles, and general cycle length that had been collected as part of their menstrual cycle history were used to estimate a cycle length and approximate ovulation day for this month. The luteal phase of the cycle was fixed at 14 days, while the follicular phase was the remaining days of their historical cycle length, since the follicular phase has been shown to vary much more between women than the luteal phase [29]. The estimated ovulation day was designated and used as their follicular phase test day. To schedule the luteal phase test day, subjects used the LH test kits starting 2 days before until 2 days after the estimated ovulation day. When they identified their LH surge with the test strips, this was considered the day of their ovulation, and the luteal phase test day was scheduled between 8 - 14 days after LH surge. Following their luteal phase test day, they were instructed to continue their menstrual cycle calendar till the onset of the next bleeding phase.

Test day protocols: Study participants were tested twice, once around the time of their ovulation (late follicular phase - FP), and once during their mid-late luteal phase (LP). On scheduled test days, the participants arrived at the test center following an overnight fast. Blood was collected first upon arrival, processed to extract serum and plasma, aliquoted and stored at $-80^{\circ} \mathrm{C}$ for further analyses.

Food Craving Inventory: During the LP test day they were given the FCI questionnaire, and asked to rate the frequency of their craving for each food over the past two weeks. The FCI has been effectively used in testretest studies without an exposure or learning effect [30]. The foods listed included items from five categories: sweets (eg: donuts, cookies, candy), high fat (eg: Alfredo sauce, cake, ice cream), starches/carbohydrates (eg: bread, pancakes, pasta, flour tortillas), fast foods (eg: hot dogs, pizza, nachos), and sweet beverages (eg: fruit smoothies, soda (diet and non-diet), sweetened coffee beverages). The participants were asked to score these foods on a five-point Likert scale of never ( 0 - once a month), to daily (6 - 7 times a week). Participants were given a definition of craving as an intense desire to consume a particular food (or food type) that is difficult to resist. A total higher score indicated higher craving of each food. Similar foods (ie) high sweet, high fat or high carbohydrate were grouped together, and scores for those foods summed in order to arrive at these food 
category scores. In this manuscript, we report on data from craving and habitual intake of high fat, high sweet

104 and high carbohydrate/starch foods.

105 Body composition: Body fat mass and fat-free mass were measured by dual energy x-ray

106 absorptiometry (DXA Hologic Discovery W, DEXA Diagnostics, Version 13.3:7) by a California

107 Department of Health Services licensed DEXA technician, either on the follicular or the luteal phase

108 test day. During the DEXA scans, the android and gynoid fat regions were determined by the Hologic

109 software.

111 Clinical markers: Fasting plasma insulin, glucose, triglycerides, total -, LDL- and HDL-cholesterol were 112 measured. Plasma insulin was measured using electro-chemiluminescence technology on a Sector Imager 2400 113 (Model 1250, Meso-Scale Discovery, Gaithersburg, MD). Plasma glucose and lipids were measured using 114 chemiluminescence technology on a Clinical Chemistry Analyser (COBAS Integra 400+, Roche Diagnostics 115 Corp., Indianapolis, IN). The fasting serum was used to measure estradiol, progesterone, dehydroepiandostrone 116 sulfate (DHEAS) and leptin. Estradiol, progesterone and leptin were measured using an electro117 chemiluminescence kit (Meso Scale Discovery) and DHEAS using chemiluminescence on the COBAS Integra 118 (Roche). Sex hormone binding globulin (SHBG) was measured using a Quantikine (R) Elisa kit (Minneapolis, $119 \mathrm{MN})$.

121 Statistical analysis: All statistical tests were conducted in R v 2.13.0 ( R-Core Team, Vienna, Austria) [31] and 122 JMP Pro 12.1 for Windows (SAS Institute, Cary NC). Normality tests using the Shapiro-Wilk test was done on 123 raw, log, square root and $\mathrm{z}$ score transformed data sets. Log transformed datasets returned normal data for all 124 variables, and this was used for the remaining analyses. All craving data reported are for LP, habitual data are 125 non-phase specific, while endocrine concentrations are phase-specific. Leptin was tested as both unadjusted and 126 body fat adjusted leptin, resulting in the same interpretations. For the sake of reporting in this manuscript, we 127 used unadjusted leptin. Linear mixed model ANOVA (using package nlme in R) with the subject as the random 
128 effect, and the phase as repeated measure was used to identify differences between follicular phase (FP) and 129 luteal phase (LP) endocrine and clinical measures. Tukey's post hoc tests were used for multiple comparisons 130 where necessary. Pearson's correlation coefficients were used to identify associations. Flexmix package [32] in $131 \mathrm{R}$ was used to evaluate the number of best fit lines that can represent the association between estradiol-leptin 132 ratio, and sweet-tasting and carbohydrate-rich food intake in this group of women. This method considers AIC 133 (Akaike information criterion, BIC (Bayesian information criteria) and ICL (Integrated Completed Likelihood). 134 This was used to determine the number of clusters to choose using a hierarchical cluster analysis (HCA). The 135 HCA was done using the Ward method with estradiol, progesterone, leptin, DHEAS, SHBG, leptin, and 136 habitual food intake and craving scores, and two clusters were identified. We used one-way ANOVA to 137 establish differences between the two clusters, and regression model comparisons to test the variable association 138 differences between the clusters. 
RESULTS

141 Seventeen women (8 Caucasian, 4 Asian, 3 African American, and 2 Hispanic) participated in this study. 142 Subject characteristics are presented in Table 1.

\section{Endocrine measures}

Progesterone was higher $(\mathrm{p}=0.0002)$ in the LP compared to FP (Figure 1), whereas estradiol was not 146 different $(\mathrm{p}=0.13)$. There was no difference in SHBG between the two phases. Overall, the estradiol and 147 progesterone profiles across the two phases resemble what is to be expected from healthy menstruating women.

148 The subjects' reports based on their urinary LH surge test matched with the FP testing day with a \pm 2 day 149 difference. The subjects also reported their next period onset, and it ranged between 1 to 6 days following the 150 LP test day. Estradiol was significantly inversely associated with leptin in FP $(r=-0.62, p=0.009)$, but no 151 association was found between estradiol and leptin in LP $(r=0.26, p=0.31)$. No significant associations 152 between progesterone and leptin, as well as progesterone and SHBG were identified.

\section{Clinical measures}

Table 2 presents all the clinical variables that were measured in LP and FP. No significant differences in 156 these parameters were found between the two phases in this study.

\section{Endocrine measures, luteal phase cravings and habitual food intake}

159 The associations between luteal phase craving and (follicular or luteal phase) endocrine measures were 160 examined separately from the associations between habitual food intake and (follicular or luteal phase) 161 endocrine measures, since the reported cravings and habitual food intake were not correlated with each other 162 (data not shown). Similarly, no differences in cognitive restraint, disinhibition or hunger based on the TFEQ 163 were found between the two phases (data not shown). 


\section{Luteal phase cravings and endocrine measures}

Figure 2 presents the significant correlations from this study. Higher carbohydrate craving in LP was reported cravings in either phase.

\section{Reported habitual food intake and endocrine measures}

Habitual intake of sweets was inversely correlated with leptin in the follicular phase $(r=-0.643, p=$ $0.005)$ and in the luteal phase $(r=-0.626, p=0.007)$. There was also a trend for intake of sweet-tasting food to be positively associated with FP SHBG $(\mathrm{r}=0.464, \mathrm{p}=0.061)$.

While estradiol was not associated with any food intake behavior, the estradiol/leptin ratio in the LP was positively associated with habitual intake of sweet-tasting foods $(r=0.583, p=0.014)$, and, although not significant, with habitual carbohydrate-rich food intake $(r=0.426, \mathrm{p}=0.088)$.

\section{Cluster analyses}

Since estradiol:leptin ratio showed an inverse relationship to that of leptin with habitual intake of sweet-tasting foods in LP, we used statistical modeling to further understand this relationship. We identified that the association between estradiol: leptin ratio and sweet-tasting foods in LP, was best fit by two regression lines, instead of one or three (Figure 3, panel A and B), indicating the likely presence of two groups of individuals, as opposed to a homogenous population. Since leptin, estradiol, progesterone and SHBG were identified as relevant endocrine variables associated with food intake and craving behaviors based on our correlational analyses, we used all these variables in a cluster analysis to determine sub-groups within our sample. We identified two clusters - cluster 1, and cluster 2, as indicated in Figure 3, Panel C. 


\section{$192 \quad$ Luteal Phase differences}

193 The differences between clusters 1 and 2 are indicated in Figure 4, 5 and 6. Cluster 1 has higher carbohydrate 194 craving $(\mathrm{p}=0.0006)$, higher estradiol $(\mathrm{p}=0.03)$ and higher sweet-tasting food craving $(\mathrm{p}=0.01)$ compared to 195 cluster 2 (Figure 4). The regression lines fitting estradiol relationship with craving sweets were different $(p=$ 196 0.005), while craving carbohydrates were not different. Estradiol: leptin and estradiol: DHEAS ratios were also 197 higher in cluster 1 compared to cluster $2(p=0.04$ and $p=0.007$ respectively) (Figure 5). Similar to estradiol 198 (Figure 6), SHBG was also higher in cluster 1 compared to cluster $2(\mathrm{p}=0.005)$. Unlike estradiol, cluster 1 had 199 a significantly different association between SHBG and craving carbohydrates compared to cluster $2(\mathrm{p}=0.05)$.

200 The relationship between SHBG and craving sweet-tasting foods was not different between clusters $(p=0.25)$.

201 Progesterone, leptin, insulin and DHEAS were not different between the two clusters. There was also no 202 significant association between progesterone, insulin or DHEAS to E2:leptin ratio. 


\section{DISCUSSION}

To our knowledge, this is the first study that examined the relationship between ovarian menstrual cycle hormones, leptin, and reported habitual food intake and craving behaviors in healthy adult women. Our study has determined that estradiol was inversely associated with leptin in the late follicular phase, but not in the mid-late luteal phase. Leptin, irrespective of phase, was inversely associated with habitual intake of sweet foods. The estradiol/leptin ratio in the luteal phase, but not the follicular phase, was positively associated with habitual intake of sweets.

Probing the leptin-estradiol relationship in the luteal phase further, two groups of women emerged, one characterized by higher craving for carbohydrates and sweet-tasting foods, higher estradiol, estradiol/leptin ratio, estradiol/DHEAS ratio, and SHBG, compared to the other. This establishes three concepts - primarily that higher plasma leptin concentration, irrespective of menstrual cycle phase, is associated with lower carbohydrate and sweet-tasting food intake. Secondly, differences in craving in the luteal phase in women may be associated with underlying endocrine differences; women with higher estradiol/ leptin ratio, or estradiol/androgen ratio and higher SHBG have greater craving for carbohydrates and sweet-tasting foods. Finally, while craving behaviors are more strongly associated with ovarian and other steroid hormones, habitual food intake is more strongly and likely independently, associated with leptin in healthy, normal weight women. In essence, there appears to be a balance between leptin and ovarian circulating hormones controlling food craving and eating behaviors (See supplemental figure 1). This balance may influence long term body weight maintenance. Further studies with overweight/obese women, or women with PMDD or polycystic ovary syndrome (PCOS) are needed to establish if the leptin-sweet food intake, estradiol/leptin ratio and food craving are disrupted, enabling unhealthy eating behaviors.

Luteal phase is associated with higher circulating progesterone, however, progesterone was not different between the two clusters, nor was it associated with SHBG. Even correcting for the effect of progesterone on SHBG (using partial Pearson's correlation), the relationship between SHBG and craving carbohydrates, and sweet rich foods remained significant (data not shown). Klump et al suggested that progesterone and estradiol interact with each other, influencing emotional eating in the mid-luteal phase [33]. 
the current report, we didn't find any evidence to support an interaction between progesterone and estradiol, SHBG, leptin, eating or craving behaviors. We only studied the women once in the follicular and luteal phase, and multiple measures throughout the menstrual cycle may be necessary to fully understand the leptin-estradiolprogesterone relationship and its impact on food intake behaviors.

Estradiol has been studied in association with food intake extensively in animals. Estradiol is anorexigenic and reduces food intake in animals. In humans however, circulating estradiol was not associated 
threshold. Binding with SHBG reduces bio-available estradiol, and hence, circulating SHBG concentrations become crucial when considering functional concentration of estradiol [39]. It is important to note that, similar to Thaler et al and Pasquali et al $[37,38]$, we are reporting total estradiol, which is a combination of free unbound estradiol ("bio-available"), as well as SHBG and albumin bound estradiol. If we had estimated or reported unbound estradiol, which is bio-available, these relationships would possibly be different. Hence SHBG, as expected, increases along with estradiol during the menstrual cycle [40]. In an interesting feedback loop, SHBG and leptin have been inversely associated with each other in women, with SHBG accounting for $\sim 18 \%$ of the variance in circulating leptin [41]. While SHBG has not been associated with specific food cravings in the past, in the present study we identified that a higher SHBG was associated with both carbohydrate-rich and sweet-tasting food cravings, in the luteal phase. We also identified that a higher SHBG available" estradiol while studying premenopausal women, especially in their luteal phase. The higher estradiol/leptin and estradiol/DHEAS ratio in cluster 1 likely indicates that in women with higher leptin (and/or androgens), the ovarian hormone influence on food intake and craving behaviors is overridden. However, in women with lower leptin or DHEAS, estradiol is associated with increased craving, especially in the luteal phase of the menstrual cycle.

A recent weight loss study that tailored the diet and lifestyle modification to the menstrual cycle found that this approach was more successful than a traditional regime that did not consider the menstrual cycle [42]. There was however, a high drop-out rate, even in the menstrual cycle diet group in this study. Personalizing a weight loss or any lifestyle diet modification, in addition to tailoring it to the menstrual cycle, also should 
281 phenotype the individual's craving behaviors and underlying endocrine milieu. Considering this craving 282 phenotype may improve adherence to and outcome of diet and lifestyle regime changes.

Limitations

285 The time when these women were tested during the follicular phase was within the range of the ovulation day as 286 we had planned it to be (i.e. \pm 2 days within ovulation), to test them at peak estradiol in circulation. However, 287 with the luteal phase testing, our time of test may have been later than we had hoped to achieve, since our 288 testing happened between 1-6 days of the next bleeding phase, indicating a later luteal phase testing than mid289 luteal phase. So, while we managed to test some of these women at mid-luteal phase, we tested some others in 290 late luteal phase. Testing all women during mid-luteal phase could have resulted in higher progesterone 291 concentrations, yielding a different association between progesterone and eating behaviors. Due to the small sample size, we may have missed observing other relationships that might exist. Also, future studies should aim to measure ovarian and other satiety hormones at multiple time points across the menstrual cycle so as to confirm these findings, and possibly reveal other endocrine - eating behavior associations.

\section{Conclusions}

297 We identified a higher estradiol, high estradiol/leptin ratio, high SHBG, and increased craving type vs a lower 298 estradiol, low estradiol/leptin ratio, low SHBG and less-craving type in women. In normal weight healthy 299 women, higher estradiol in the luteal phase is associated with increased craving for sweet-tasting and 300 carbohydrate rich foods. We believe the estradiol-leptin relationship is a defining quality in whether or not 301 women crave sweet-tasting and carbohydrate rich foods. Studies like this generate knowledge of endocrine302 eating behavior relationships that can help women understand the physiological basis of their cravings. A 303 woman's propensity to crave certain foods may be used as an educational tool to advocate healthier options, 304 especially as it relates to high carbohydrate, high sweet foods, since they are calorically dense. Future studies 305 should focus on replicating, as well as probing these associations in overweight/obese, or women with PCOS, 
307 body weight maintenance. Relationships between ovarian hormones and other metabolic health parameters may 308 also come into light from linking eating behaviors with metabolic disease risk. 


\section{REFERENCES}

311 [1] Hormes, J. M., Timko, C. A. All cravings are not created equal. Correlates of menstrual versus non-cyclic 312 chocolate craving. Appetite. 2011,57:1-5.

313 [2] Bryant, M., Truesdale, K. P., Dye, L. Modest changes in dietary intake across the menstrual cycle:

314 implications for food intake research. The British journal of nutrition. 2006,96:888-94.

315 [3] McNeil, J., Doucet, E. Possible factors for altered energy balance across the menstrual cycle: a closer look at 316 the severity of PMS, reward driven behaviors and leptin variations. European journal of obstetrics, gynecology, 317 and reproductive biology. 2012,163:5-10.

318 [4] Barr, S. I., Janelle, K. C., Prior, J. C. Energy intakes are higher during the luteal phase of ovulatory 319 menstrual cycles. The American journal of clinical nutrition. 1995,61:39-43.

320 [5] Yen, J. Y., Chang, S. J., Ko, C. H., Yen, C. F., Chen, C. S., Yeh, Y. C., et al. The high-sweet-fat food 321 craving among women with premenstrual dysphoric disorder: emotional response, implicit attitude and rewards 322 sensitivity. Psychoneuroendocrinology. 2010,35:1203-12.

323 [6] Gorczyca, A. M., Sjaarda, L. A., Mitchell, E. M., Perkins, N. J., Schliep, K. C., Wactawski-Wende, J., et al. 324 Changes in macronutrient, micronutrient, and food group intakes throughout the menstrual cycle in healthy, 325 premenopausal women. European journal of nutrition. 2015.

326 [7] Potenza, M. N., Grilo, C. M. How Relevant is Food Craving to Obesity and Its Treatment? Frontiers in 327 psychiatry. 2014,5:164.

328 [8] Dye, L., Blundell, J. E. Menstrual cycle and appetite control: implications for weight regulation. Human 329 reproduction. 1997,12:1142-51.

330 [9] Yu, Z., Geary, N., Corwin, R. L. Individual effects of estradiol and progesterone on food intake and body 331 weight in ovariectomized binge rats. Physiology \& behavior. 2011,104:687-93.

332 [10] Fungfuang, W., Terada, M., Komatsu, N., Moon, C., Saito, T. R. Effects of estrogen on food intake, serum 333 leptin levels and leptin mRNA expression in adipose tissue of female rats. Laboratory animal research. $334 \quad 2013,29: 168-73$. 
[11] Butera, P. C. Estradiol and the control of food intake. Physiology \& behavior. 2010,99:175-80.

[12] Santollo, J., Katzenellenbogen, B. S., Katzenellenbogen, J. A., Eckel, L. A. Activation of ERalpha is necessary for estradiol's anorexigenic effect in female rats. Hormones and behavior. 2010,58:872-7.

[13] Gray, J. M., Wade, G. N. Food intake, body weight, and adiposity in female rats: actions and interactions of progestins and antiestrogens. The American journal of physiology. 1981,240:E474-81.

[14] Curtis, K. S., Stratford, J. M., Contreras, R. J. Estrogen increases the taste threshold for sucrose in rats. Physiology \& behavior. 2005,86:281-6.

[15] Kubasova, N., Burdakov, D., Domingos, A. I. Sweet and Low on Leptin: Hormonal Regulation of Sweet Taste Buds. Diabetes. 2015,64:3651-2.

[16] Macedo, D. M., Diez-Garcia, R. W. Sweet craving and ghrelin and leptin levels in women during stress. Appetite. 2014,80:264-70.

[17] Karhunen, L. J., Lappalainen, R. I., Haffner, S. M., Valve, R. H., Tuorila, H., Miettinen, H., et al. Serum leptin, food intake and preferences for sugar and fat in obese women. International journal of obesity and related metabolic disorders : journal of the International Association for the Study of Obesity. 1998,22:819-21. [18] Ajala, O. M., Ogunro, P. S., Elusanmi, G. F., Ogunyemi, O. E., Bolarinde, A. A. Changes in serum leptin during phases of menstrual cycle of fertile women: relationship to age groups and fertility. International journal of endocrinology and metabolism. 2013,11:27-33.

[19] Mannucci, E., Ognibene, A., Becorpi, A., Cremasco, F., Pellegrini, S., Ottanelli, S., et al. Relationship between leptin and oestrogens in healthy women. European journal of endocrinology / European Federation of Endocrine Societies. 1998,139:198-201.

[20] Gambino, Y. P., Maymo, J. L., Perez-Perez, A., Duenas, J. L., Sanchez-Margalet, V., Calvo, J. C., et al. 17Beta-estradiol enhances leptin expression in human placental cells through genomic and nongenomic actions. Biology of reproduction. 2010,83:42-51.

[21] Pantaleao, T. U., Mousovich, F., Rosenthal, D., Padron, A. S., Carvalho, D. P., da Costa, V. M. Effect of serum estradiol and leptin levels on thyroid function, food intake and body weight gain in female Wistar rats. Steroids. 2010,75:638-42. 
[22] Pelleymounter, M. A., Baker, M. B., McCaleb, M. Does estradiol mediate leptin's effects on adiposity and 362 body weight? The American journal of physiology. 1999,276:E955-63.

363 [23] Pliner, P., Fleming, A. S. Food intake, body weight, and sweetness preferences over the menstrual cycle in 364 humans. Physiology \& behavior. 1983,30:663-6.

365 [24] Davidsen, L., Vistisen, B., Astrup, A. Impact of the menstrual cycle on determinants of energy balance: a 366 putative role in weight loss attempts. International journal of obesity. 2007,31:1777-85.

367 [25] Wetherill, R. R., Franklin, T. R., Allen, S. S. Ovarian hormones, menstrual cycle phase, and smoking: a 368 review with recommendations for future studies. Current addiction reports. 2016,3:1-8.

369 [26] Study of Women Across the Nation, Study Forms. 2012.

370 [27] White, M. A., Whisenhunt, B. L., Williamson, D. A., Greenway, F. L., Netemeyer, R. G. Development and 371 validation of the food-craving inventory. Obes Res. 2002,10:107-14.

372 [28] Stunkard, A. J., Messick, S. The three-factor eating questionnaire to measure dietary restraint, disinhibition 373 and hunger. Journal of psychosomatic research. 1985,29:71-83.

374 [29] Cole, L. A., Ladner, D. G., Byrn, F. W. The normal variabilities of the menstrual cycle. Fertility and $375 \quad$ sterility. 2009,91:522-7.

376 [30] Anton, S. D., Gallagher, J., Carey, V. J., Laranjo, N., Cheng, J., Champagne, C. M., et al. Diet type and 377 changes in food cravings following weight loss: findings from the POUNDS LOST Trial. Eating and weight 378 disorders : EWD. 2012,17:e101-8.

379 [31] Team., R. D. C. R: A language and environment for statistical computing. R Foundation for Statistical 380 Computing. Vienna, Austria.2010.

381 [32] Leisch, F. FlexMix: A General Framework for Finite Mixture Models and Latent Class Regression in R. $382 \quad$ Journal of Statistical Software. 2004,11.

383 [33] Klump, K. L., Keel, P. K., Racine, S. E., Burt, S. A., Neale, M., Sisk, C. L., et al. The interactive effects of 384 estrogen and progesterone on changes in emotional eating across the menstrual cycle. Journal of abnormal $385 \quad$ psychology. 2013,122:131-7. 
[34] Oh, H., Smith-Warner, S. A., Tamimi, R. M., Wang, M., Xu, X., Hankinson, S. E., et al. Dietary Fat and 387 Fiber Intakes Are Not Associated with Patterns of Urinary Estrogen Metabolites in Premenopausal Women. J $388 \quad$ Nutr. 2015,145:2109-16.

389 [35] Klump, K. L., Racine, S. E., Hildebrandt, B., Burt, S. A., Neale, M., Sisk, C. L., et al. Ovarian Hormone 390 Influences on Dysregulated Eating: A Comparison of Associations in Women with versus without Binge 391 Episodes. Clin Psychol Sci. 2014,2:545-59.

392 [36] Selby, C. Sex hormone binding globulin: origin, function and clinical significance. Annals of clinical 393 biochemistry. 1990,27 ( Pt 6):532-41.

394 [37] Pasquali, R., Vicennati, V., Bertazzo, D., Casimirri, F., Pascal, G., Tortelli, O., et al. Determinants of sex 395 hormone-binding globulin blood concentrations in premenopausal and postmenopausal women with different 396 estrogen status. Virgilio-Menopause-Health Group. Metabolism: clinical and experimental. 1997,46:5-9. 397 [38] Thaler, M. A., Seifert-Klauss, V., Luppa, P. B. The biomarker sex hormone-binding globulin - from 398 established applications to emerging trends in clinical medicine. Best practice \& research. Clinical 399 endocrinology \& metabolism. 2015,29:749-60.

400 [39] Rosner, W. Plasma steroid-binding proteins. Endocrinology and metabolism clinics of North America. $401 \quad 1991,20: 697-720$. 402 [40] Plymate, S. R., Moore, D. E., Cheng, C. Y., Bardin, C. W., Southworth, M. B., Levinski, M. J. Sex 403 hormone-binding globulin changes during the menstrual cycle. J Clin Endocrinol Metab. 1985,61:993-6. 404 [41] Milewicz, T., Krzysiek, J., Janczak-Saif, A., Sztefko, K., Krzyczkowska-Sendrakowska, M. Age, insulin, 405 SHBG and sex steroids exert secondary influence on plasma leptin level in women. Endokrynologia Polska. $406 \quad 2005,56: 883-90$.

407 [42] Geiker, N. R., Ritz, C., Pedersen, S. D., Larsen, T. M., Hill, J. O., Astrup, A. A weight-loss program 408 adapted to the menstrual cycle increases weight loss in healthy, overweight, premenopausal women: a 6-mo 409 randomized controlled trial. Am J Clin Nutr. 2016. 
Figure Legends

Figure 1: Serum estradiol, progesterone, DHEAS and SHBG measured during follicular (FP) and luteal phase (LP) of the menstrual cycle (mean \pm SEM). Progesterone was higher in LP than during FP $(\mathrm{p}<0.001)$; Estradiol, DHEAS and SHBG were not different between the two phases. Leptin was inversely associated with estradiol in the follicular phase $(r=-0.62, p=0.009)$, but not the luteal phase $(r=0.26, p=0.31)$

Figure 2: Correlation analysis results of habitual food intake or luteal phase craving (x axis) and endocrine measures (y axis), with the Pearson's correlation coefficient $r$ value and corresponding $p$ value. Significant positive correlations are in red and inverse correlations are in green. Significant correlations were identified at $\mathrm{p}<0.05$. E2:leptin ratio $=$ estradiol leptin ratio.

Figure 3: Panel A and B: Flexmix indication of number of clusters in dataset using estradiol and sweet-rich food intake. AIC (Akaike information criterion, BIC (Bayesian information criteria) and ICL (Integrated Completed Likelihood) indicated using different colors are used to measure the number of best fit lines. Testing for 2 clusters (Panel A) did not arrive at a single response while testing for 3 components indicated that AIC,

427 BIC and ICL indicate two clusters, chosen by the lowest scores (Panel B). Panel C: Dendrogram presents output from hierarchical clustering using estradiol, progesterone, leptin, SHBG and food intake and craving behavior data from all 17 volunteers using the Ward method. Numbers on the left indicate subject ID numbers. In panel C, Clusters are shown in Red (cluster 1) and Green numbering (cluster 2).

Figure 4: Estradiol, sweet and carbohydrate craving scores in the mid-late luteal phase were significantly higher in cluster 1 , compared to cluster 2 (indicated by '*'; $p=0.03,0.01$ and 0.001 , respectively - top panels). The bars represent group means and error bars are standard error of the mean. The bottom panels depict the 
435 associations between estradiol and sweet cravings for clusters 1 and 2 (left) and estradiol and carbohydrate

436 craving for the two clusters (right). Comparison of regression lines for cluster 1 versus cluster 2 showed that the 437 relationship between estradiol and craving sweet foods was different $(p=0.005)$ between cluster 1 and 2 , 438 whereas the relationship between estradiol and craving carbohydrate-rich foods was not different between $439 \quad$ clusters $(p=0.279)$.

440

441 Figure 5: Estradiol: leptin and estradiol:DHEAS ratios were higher in Cluster 1 than Cluster 2 ( $p=0.04$ and $442 \mathrm{p}=0.007$ respectively). Bars represent group means and error bars are standard error of the mean.

443

444 Figure 6: SHBG was significantly higher in cluster 1 than 2 (top bar graph, $p=0.005$ ). The bars represent 445 group means and error bars are standard error of the mean. The bottom panels depict the association between 446 SHBG and craving sweet foods (left) and SHBG and craving carbohydrate-rich foods (right).Comparison of 447 regression lines for cluster 1 versus cluster 2 showed that the relationship between SHBG and craving 448 carbohydrate-rich foods was different $(\mathrm{p}=0.05)$ between clusters, but the regression lines for SHBG versus 449 craving sweet foods were not different between clusters $(\mathrm{p}=0.25)$. 

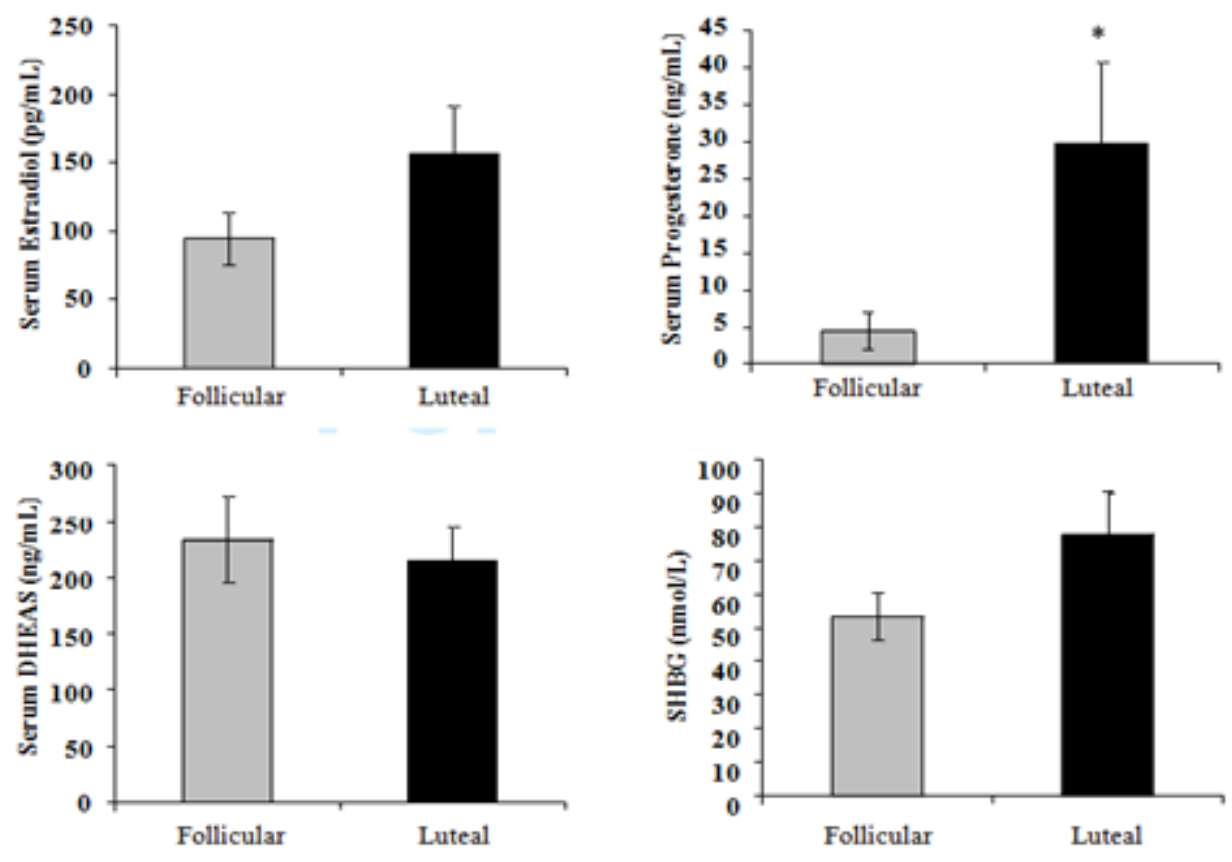

451

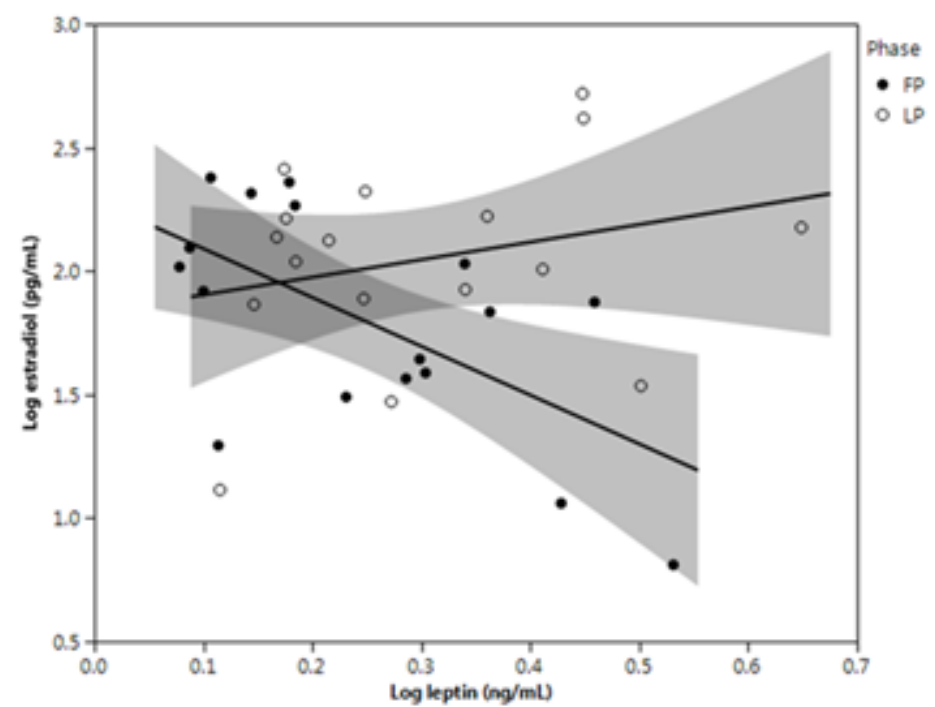

452 Figure 1: Serum estradiol, progesterone, DHEAS and SHBG measured during follicular (FP) and luteal 453 phase (LP) of the menstrual cycle (mean \pm SEM). Progesterone was higher in LP than during FP $454 \quad(\mathrm{p}<0.001)$; Estradiol, DHEAS and SHBG were not different between the two phases. Leptin was 455 inversely associated with estradiol in the follicular phase $(r=-0.62, p=0.009)$, but not the luteal phase $456 \quad(\mathrm{r}=0.26, \mathrm{p}=0.31)$ 

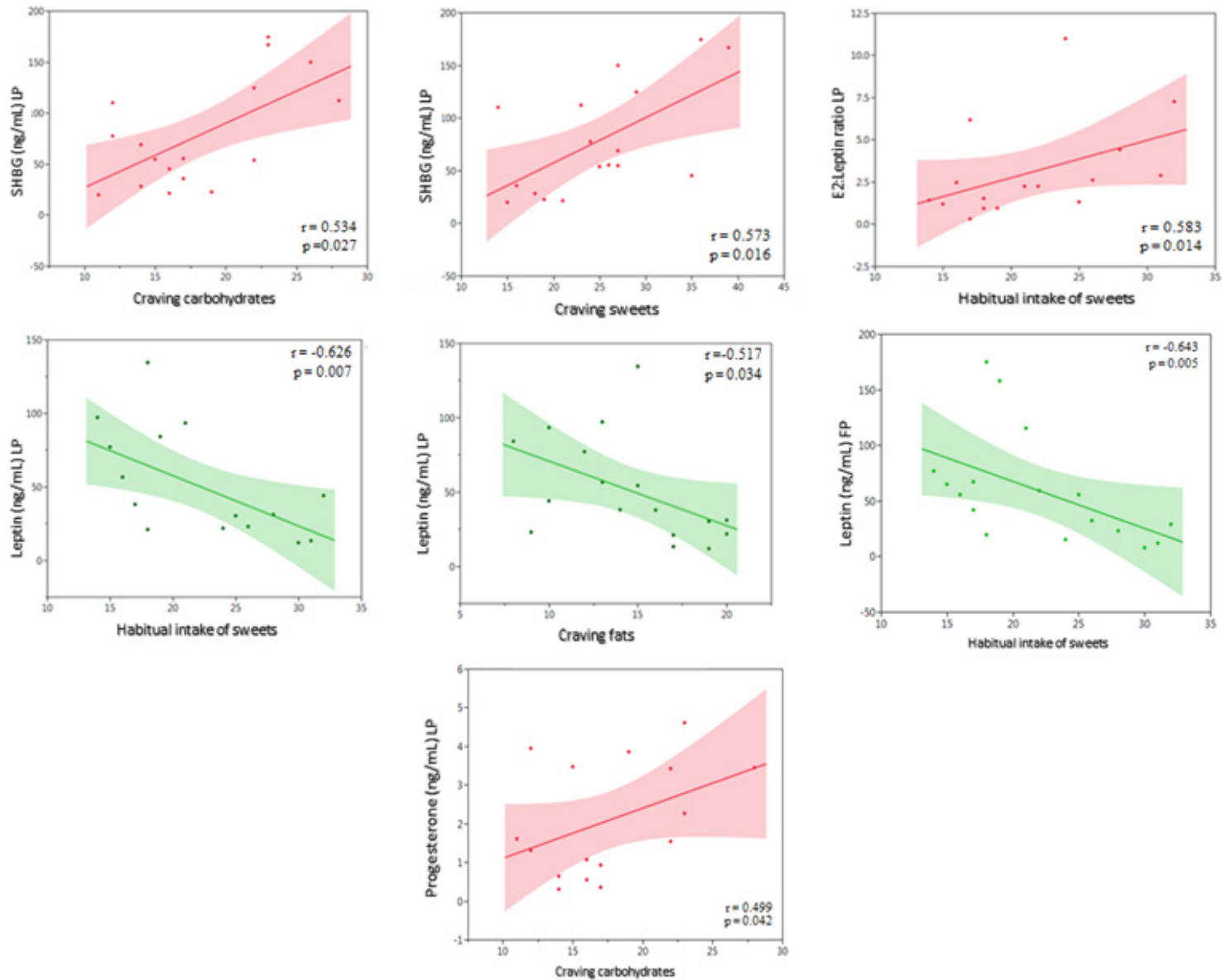

Figure 2: Correlation analysis results of habitual food intake or luteal phase craving ( $\mathrm{x}$ axis) and endocrine measures (y axis), with the Pearson's correlation coefficient $r$ value and corresponding $\mathrm{p}$ value. Significant positive correlations are in red and inverse correlations are in green. Significant correlations were identified at $\mathrm{p}<0.05$. E2:leptin ratio $=$ estradiol leptin ratio. 


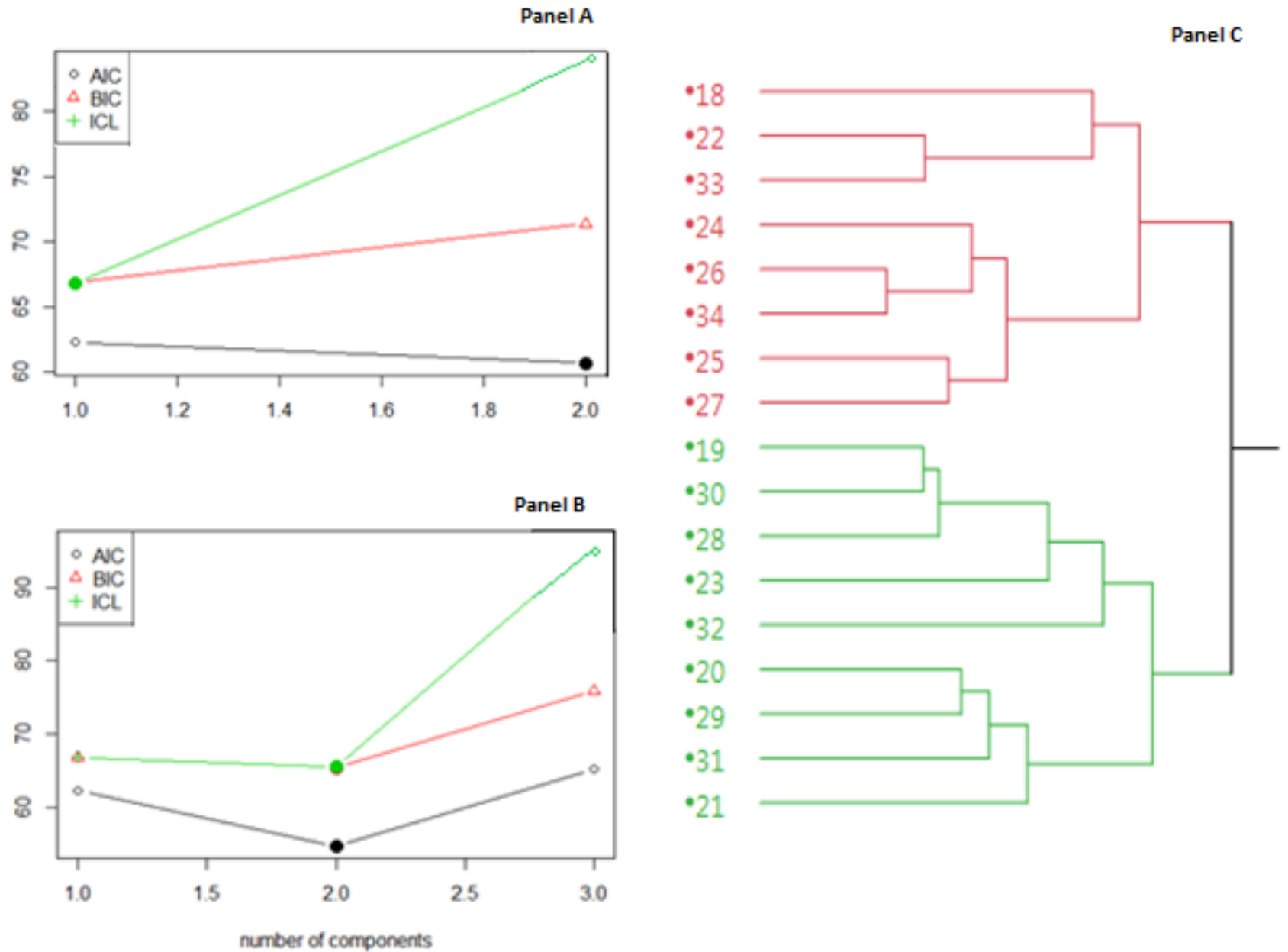

459

Figure 3: Panel A and B: Flexmix indication of number of clusters in dataset using estradiol and sweet-rich food intake. AIC (Akaike information criterion, BIC (Bayesian information criteria) and ICL (Integrated Completed Likelihood) indicated using different colors are used to measure the number of best fit lines. Testing for 2 clusters (Panel A) did not arrive at a single response while testing for 3 components indicated that AIC, BIC and ICL indicate two clusters, chosen by the lowest scores (Panel B). Panel C: Dendrogram presents output from hierarchical clustering using estradiol, progesterone, leptin, SHBG and food intake and craving behavior data from all 17 volunteers using the Ward method. Numbers on the left indicate subject ID numbers. In panel C, Clusters are shown in Red (cluster 1) and Green numbering (cluster 2). 

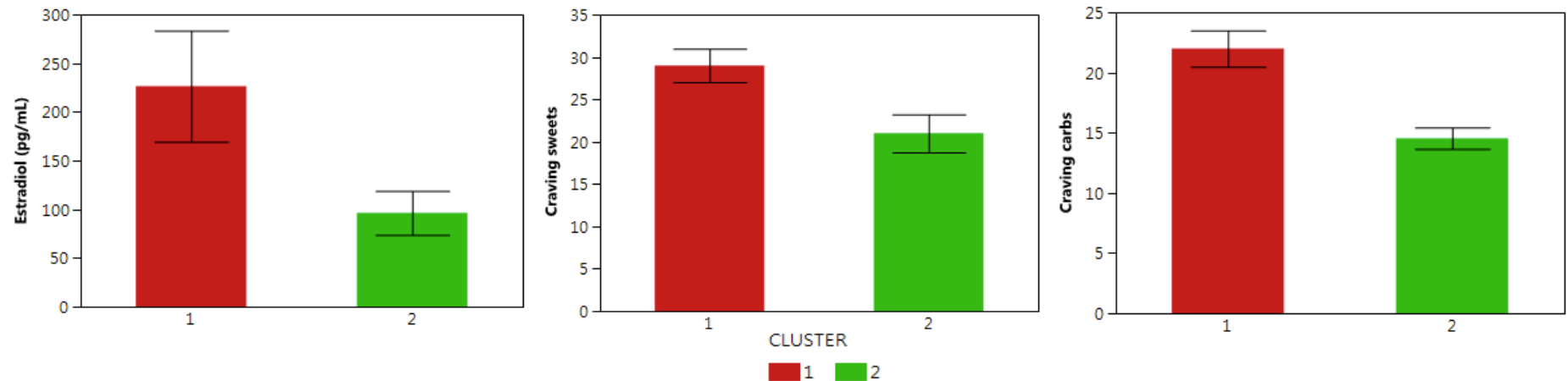

466
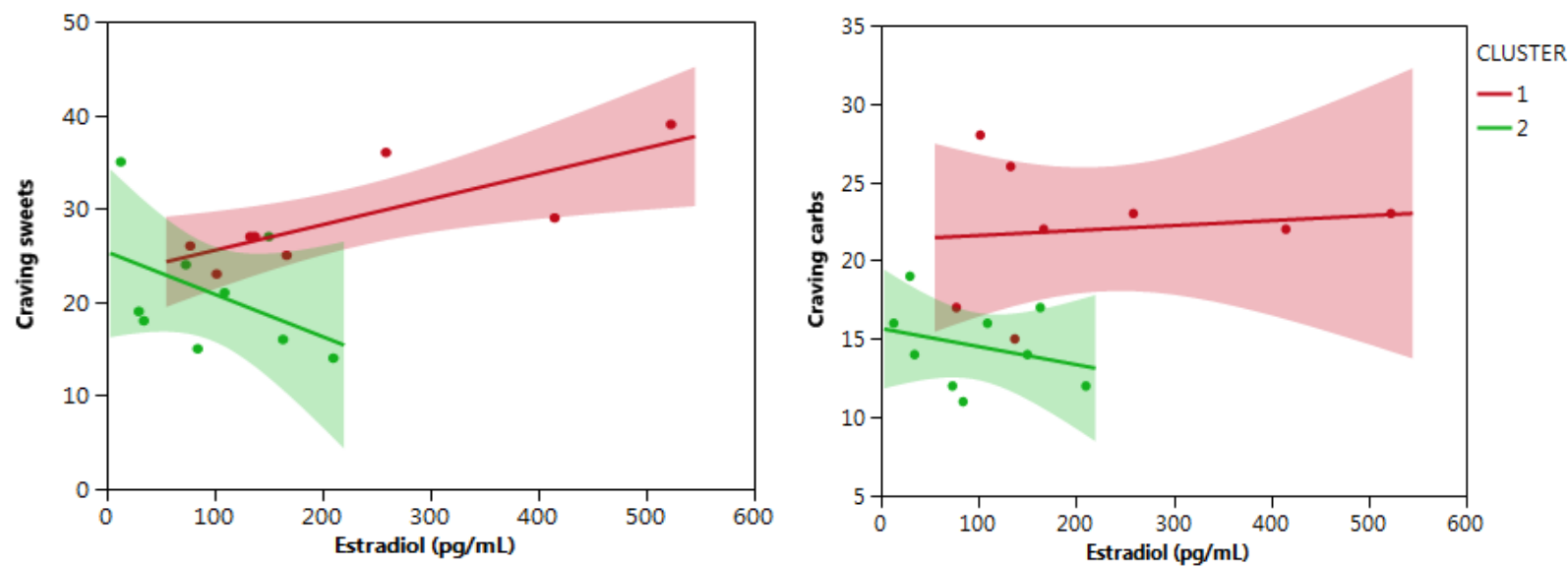

Figure 4: Estradiol, sweet and carbohydrate craving scores in the mid-late luteal phase were significantly higher in cluster 1 , compared to cluster 2 (indicated by '*'; $p=0.03,0.01$ and 0.001 , respectively - top panels). The bars represent group means and error bars are standard error of the mean. The bottom panels depict the associations between estradiol and sweet cravings for clusters 1 and 2 (left) and estradiol and carbohydrate craving for the two clusters (right). Comparison of regression lines for cluster 1 versus cluster 2 showed that the relationship between estradiol and craving sweet foods was different $(\mathrm{p}=0.005)$ between cluster 1 and 2 , whereas the relationship between estradiol and craving carbohydrate-rich foods was not different between clusters $(p=0.279)$. 

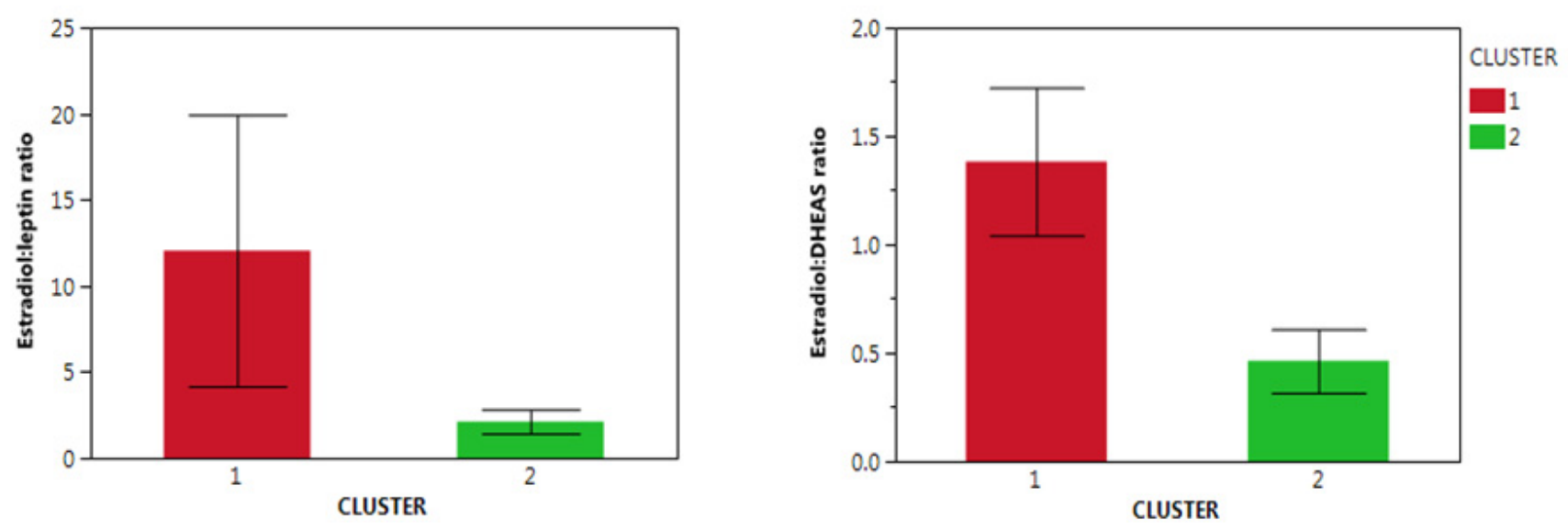

Figure 5: Estradiol: leptin and estradiol:DHEAS ratios were higher in Cluster 1 than Cluster $2(\mathrm{p}=0.04$ and $\mathrm{p}=0.007$ respectively). Bars represent group means and error bars are standard error of the mean. 

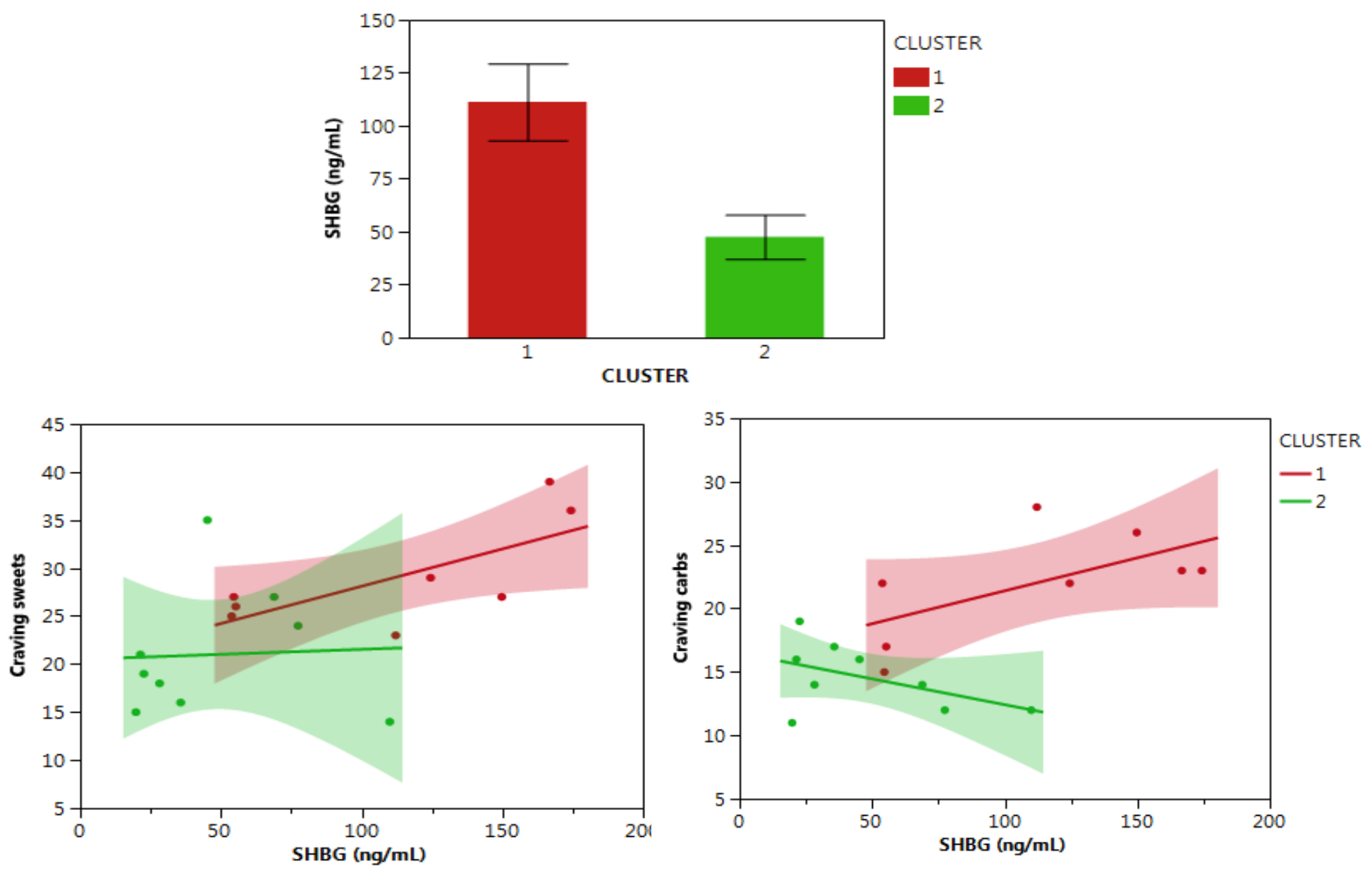

Figure 6: SHBG was significantly higher in cluster 1 than 2 (top bar graph, $p=0.005$ ). The bars represent group means and error bars are standard error of the mean. The bottom panels depict the association between SHBG and craving sweet foods (left) and SHBG and craving carbohydrate-rich foods (right).Comparison of regression lines for cluster 1 versus cluster 2 showed that the relationship between SHBG and craving carbohydrate-rich foods was different $(\mathrm{p}=0.05)$ between clusters, but the regression lines for SHBG versus craving sweet foods were not different between clusters $(\mathrm{p}=0.25)$. 
Table 1: Characteristics of study participants $(n=17)$.

\begin{tabular}{lc}
\hline Parameter & Mean \pm SD \\
\hline Age $(\mathrm{y})$ & $23.2 \pm 4.8$ \\
Weight $(\mathrm{kg})$ & $61.3 \pm 7.8$ \\
BMI $\left(\mathrm{kg} / \mathrm{m}^{2}\right)$ & $30.4 \pm 2.6$ \\
Cycle length $(\mathrm{d})$ & $27.9 \pm 4.9$ \\
Total Body fat $(\%)$ & $16.9 \pm 4.0$ \\
Total fat mass $(\mathrm{kg})$ & $24.2 \pm 6.8$ \\
Android fat $(\%)$ & $34.9 \pm 5.4$ \\
\hline Gynoid fat $(\%)$ & \\
\hline
\end{tabular}


Table 2: Fasting measures of insulin, glucose, lipid profile and insulin sensitivity and resistance estimates.

\begin{tabular}{lcc}
\hline Parameter & Follicular phase & Luteal phase \\
\hline Insulin (mU/mL) & $5.3 \pm 0.9$ & $9.4 \pm 3.7$ \\
Leptin $(\mathrm{ng} / \mathrm{mL})$ & $51.0 \pm 34.9$ & $59.3 \pm 49.0$ \\
Glucose (mg/dL) & $88.0 \pm 1.1$ & $87.9 \pm 1.3$ \\
Triglycerides (mg/dL) & $69.6 \pm 6.6$ & $154.1 \pm 5.9$ \\
Total Cholesterol (mg/dL) & $151.9 \pm 6.5$ & $61.6 \pm 2.7$ \\
HDL-c (mg/dL) & $61.2 \pm 2.3$ & $78.8 \pm 4.9$ \\
LDL-c (mg/dL) & $76.8 \pm 5.8$ & $2.0 \pm 0.8$ \\
HOMA-IR & $1.1 \pm 0.2$ & $0.40 \pm 0.02$ \\
QUICKI & $0.41 \pm 0.02$ & \\
\hline
\end{tabular}

No differences were found between the phases 